\title{
A Cross-layer Scheduling Algorithm Based on Cognitive Radio Network
}

\author{
Changpeng $\mathrm{Ji}^{1}$, Xiaoqiu Nie ${ }^{2}$ and Yujie Yuan ${ }^{3}$ \\ ${ }^{1}$ School of Electronics and Information Engineering, Liaoning Technical University, Huludao 125105 P.R. China \\ ${ }^{2}$ Institute of Graduate, Liaoning Technical University, Huludao 125105 P.R. China \\ ${ }^{3}$ Institute of Graduate, Liaoning Technical University, Huludao 125105 P.R. China
}

Received: 18 Oct. 2012, Revised: 5 Feb. 2013, Accepted: 7 Feb. 2013

Published online: 1 Jun. 2013

\begin{abstract}
Cognitive radio network users have different QoS requirements. In order to provide different QoS requirements to users as well as increase the capacities of the cognitive radio networks, this paper presents a first in-depth comparative study on different factors effect QoS which include system performance, power interference, wireless channel conditions, services priorities and fairness constraints. A cross-layer scheduling algorithm which could optimize system performance and control power interference is proposed. Latency default probability of real-time users and throughput performance of non- real-time users are respectively used to measure the fairness of real-time operational system and non-real-time operational system. Simulation results are computed and the results are shown. Delay performance of real-time traffic and throughput performance of non-real-time traffic are guaranteed by setting reasonable weight to index. And power interference to authorized users caused by cognitive radio network users reduces obviously. And system obtains a very good fair performance.
\end{abstract}

Keywords: Cognitive Radio Network, cross-layer scheduling algorithm, power interference, system performance, QoS

\section{Introduction}

As the rapid development and application of wireless communication technology, wireless spectrum resource has become more and more frequent [1]. However, the lack of wireless spectrum is a limitation to the development of wireless communication. At the same time, because of the fixed allocation of wireless spectrum, spectrum resources can not be used efficiently. In order to solve the problem of low utilization of spectrum resources, it is very important for us to find an efficient way to improve the wireless spectrum resources utilization ratio. Cognitive Radio (CR) technology is considered to be the best solution to solve the problem that low utilization ratio of the wireless spectrum. CR technology provides a strong technical support for easing the shortage of spectrum resources, dynamic frequency spectrum management and improving utilization ratio of wireless spectrum [2]. The conception of Cognitive Radio Network (CRN) was proposed by Motorola and Virginia Tech at first on the basis of CR [1].

In addition, with the development of wireless communication technology, the drawback of traditional layered structure protocol design is fully disclosed. Cross-layer design has been widely recognized, and already becomes a key technology for next-generation wireless communication technology. Traditional layered structure protocol design is very successful for wired network. But owing to accessing conflict, interference between users, signal fading and other environmental factors in wireless network are more serious than wired network. Two important problems exist in traditional layered structure protocol design; they can be summarized as follows [3]:

1) Non-optimal. Traditional layered structure protocol design does not allow to share information between the different layers. So information in each layer is not abundant. Traditional layered structure protocol design cannot ensure performance optimization for entire network.

2) Not flexible. In traditional layered structure protocol, network requires running in the worst case. So it cannot adapt to the change of environment, which leads to the existing protocol stack cannot adapt to changes in the wireless mobile environment flexibility. And the protocol

\footnotetext{
*Corresponding author e-mail: ccp@lntu.edu.cn
} 
stack cannot make full use of terminate spectrum resources and power resource.

To solve the two problems proposed above, cross-layer scheduling mechanism is introduced into wireless network. It makes global adaptive changes based on application requirements and network conditions, which improves performance of the whole system $[4,5]$.

In fact, the concept of cross-layer design of wireless communication quickly attracted a widely attention in academic and industry since it is proposed. And it becomes an important part in standards for future communication network. Currently, there are many mature cross-layer scheduling algorithms such as classic Proportional Fair Scheduling (PFS) algorithm, Maximum Signal to Noise Ratio (Max SNR) algorithm and so on. But in these algorithms, the effects to QoS caused by different factors such as system performance, power interference, and wireless channel conditions and so on did not take consideration. In this view, based on the deeply analysis of cognitive radio network architecture, a novel cross-layer scheduling algorithm is proposed in this paper. It makes full use of MAC layer queue status information [6] and quality of service (QoS) state information, and no need to change network configuration. The scheduling algorithm achieves to guarantee cognitive users who do not have significant degradation in QoS performance. At the same time, it significantly reduces the power interference to authorized users caused by cognitive users.

This paper is organized as follows. In Section 2, it introduces the system model. In Section 3, QoS criticality, mixed fairness criteria and scheduling algorithm are proposed. In Section 4, it shows the performance of our scheduling algorithm by simulations. Finally, the conclusions are made in Section 5.

\section{System Model}

The architecture of network system is shown in Fig.1. There are a number of transceivers that are used for transmitting data in CRN, base station corresponding to the receiver, and CPE corresponding to the transmitter. Besides data reception, the base stations of CRN are also responsible for controlling the information collection and broadcasting. That is because this scenario corresponds to the standard of the uplink data transfer mode. Authorization system is radio system or TV system which works in authorized VHF/UHF band. According to its geographical location and distance from authorization system, CRN achieves the spectrum reuse in space by power control.

For convenience, it assumes that power interference made by access system cognitive users to authorized users is all meets the interference temperature(IT) limit [6]. IT is used to measure and manage the cognitive users' power interference. In a particular band, cognitive users are allowed to access to network if interference caused by

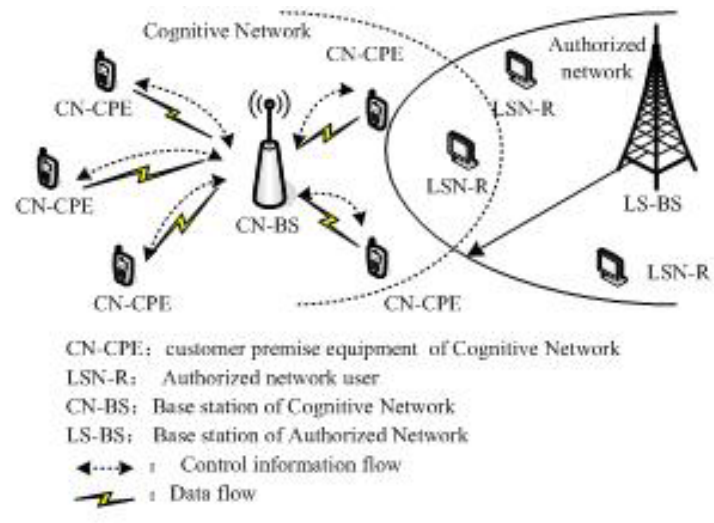

Fig. 1 Architecture of network system.

cognitive users do not exceed the interference temperature threshold. IT can be regarded as a power interference upper limit for cognitive users allowed access to licensed spectrum. Point-to-multipoint (PMP) topology is used in CRN, and the system has a base station and multiple user stations. The communication manner between cognitive users and CRN base station is time division multiplexing/time division multiple access ways, and communicate through the wireless channel. Each frame in the wireless channel is considered to be quasi-static, that is to say channel state in the duration of each frame remains the same, but it may change in different frames. At the same time, CRN base stations obtain information of each channel link by feedback information. Furthermore, the physics layer of cognitive users utilizes adaptive Modulation and Coding (AMC) to improve performance. The processing unit in physical layer of CRN is frame. The frame is divided into $N_{c}+N_{d}$ slots through time-division multiplexing, where $N_{c}$ slots are used to carry control information and pilot information, $N_{d}$ slots are used to transmit data. The processing unit in media access control (MAC) layer is data packet [7]. Each data packet includes a fixed $N_{b}$ bits which includes header, payload, cyclic redundancy check (CRC). It can be seen in Ref.[8], the transmission rate is $R_{n}$ bits/symbol when selects mode $n$ for data transmission, so there are $\frac{N_{b}}{R_{n}}$ symbols in each data packet. For convenience, the number of symbols in each slot is $\frac{2 N_{b}}{R_{1}}$ symbols, $R_{1}$ is transmission rate when selects mode 1 for data transmission. So each slot can transfer $\frac{2 R_{n}}{R_{1}}$ data packets when mode $n$ is choose for transmission. 


\section{Algorithm Descriptions}

In CRN, maximum waiting delay of packets in real-time traffic cannot exceed tolerable delay. That is to say:

$$
D_{i} \geq W_{i}(t)
$$

Where $D_{i}$ is maximum tolerable delay of connection $i$, $W_{i}(t)$ is head of the queue waiting latency of connection $i$ at time $t$.

Non-real-time traffic needs to ensure average transfer rate is not less than the minimum guaranteed rate, which:

$$
\frac{T_{i}(t)}{T_{i}} \geq 1
$$

Where, $T_{i}$ is minimum guaranteed rate requirements of connection $i, T_{i}(t)$ is average transfer rate of connection $i$ at time $t, T_{i}(t)$ can be updated at each moment by the smoothing process in equation (3)[9]:

$$
T_{i}(t+1)=\left\{\begin{array}{l}
T_{i}(t)\left(1-1 / t_{c}\right),\left(i \neq i^{*}\right) \\
T_{i}(t)\left(1-1 / t_{c}\right)+N_{d} \times R_{i}(t) / t_{c},\left(i=i^{*}\right)
\end{array}\right.
$$

Where, $i^{*}$ is the connection that obtains the opportunity for being scheduled at time $t, t_{c}$ is the size of sliding window.

Different types of traffic have different minimum QoS requirements [10]. Real-time traffic is to satisfy the minimum QoS requirements by ensuring waiting time of packets in the queue of connection $i$ do not exceed the maximum tolerable delay. Non-real-time traffic is to satisfy the minimum QoS requirements by ensuring average transfer rate of connection $i$ is greater than the minimum guaranteed rate.

In summary, QoS guarantee function of connection $i$, $S_{i}(t)$ can be defined as (4):

$$
S_{i}(t)=\left\{\begin{array}{l}
D_{i}-W_{i}(t), i \in R T \\
\frac{T_{i}(t)\left(1-1 / t_{c}\right)}{T_{i}}, i \in N R T
\end{array}\right.
$$

For real-time traffic, $S_{i}(t)$ describes the distance between the waiting time and the maximum tolerable. For non-real-time traffic, average transfer rate $T_{i}(t)$ need not be less than the minimum guaranteed rate of $T_{i}$. Thus, $S_{i}(t)<1$ means that the QoS state of connection $i$ will violate minimum QoS requirements. Connection $i$ needs to set a higher priority to ensure that it can be served as soon as possible.

Fairness performance among users is an important criterion to measure scheduling algorithm [11]. System performance only can be optimized simple without a good fairness criterion. For example, Max SNR algorithm will make the user who has the best channel conditions or nearest from the base station acquires most opportunity for scheduling. But the user who has bad channel conditions or far from the base station does not get the chance for scheduling, which makes the performance worse and it will make users dose not satisfy with the services provided by the system, or even out of service. A lot of literatures have made a deep study on fairness criteria [12]. But some of them only adjust to single service type.

It is difficult for the system which has many different types of traffic coexist to design the mix fairness measure criteria. This is because different types of traffic have their different traffic characteristics. For example, the arrival process of real-time traffic data is generally assumed to obey a distribution function, but the arrival process of non-real-time traffic data is generally assumed to be always available, which in essence is not fair to real-time traffic when the channel access of each connection be taken into consideration. Because it is not always has packet of real-time traffic waiting for being service in the queue all the time. So it does not require occupying the channel prolonged. On the other hand, different types of users are not concerned the same QoS parameters. Such as real-time traffic is more concerned about delay performance, and non-real-time traffic is more concerned about the throughput performance. Their perception on fairness is largely depends on QoS performance. You cannot acquire an accurately description for fairness performance of different users if you simply use the same parameter to measure different types of traffic. Such as in the case of fairness throughput criteria, because most real-time users are not concerned about QoS performance rather than throughput, which makes it cannot accurately reflect the fairness perception of real-time users. And it is not reasonable for the system fairness performance [13].

To solve problems that exist in the fairness criteria of different types of traffic, a mixed fairness criteria is proposed in this paper. For the different characteristics of different types of traffic, different fairness factors are designed. For example, packet average waiting time is designed for real-time traffic, and transmission rate is used for measuring fair perception for non-real-time traffic. At the same time, different weights are used by different connection in the process of scheduling to provide a relatively fair performance between different types of traffic.

The fair factor $f_{i}(t)$ can be defined as:

$$
f_{i}(t)=\left\{\begin{array}{l}
\frac{\min _{j \in R T}\left\{a \omega_{j}(t)\right\}}{a \omega_{i}(t)}, i \in R T \\
\frac{T_{i}(t)}{\max _{j \in N R T}\left\{T_{j}(t)\right\}}, i \in N R T
\end{array},\right.
$$

Where, $a \omega_{i}(t)$ is average packet waiting time of connection $i$ at time $t$, it can be calculated as follows:

$$
a \omega_{i}(t)=\frac{1}{N} \sum_{k=1}^{N} p \omega_{k}(t)
$$


Where, $p \omega_{k}(t)$ is waiting time of $k$-th packet in connection $i$. According to the send time and the arrival time of each packet, you can simply calculate the values of $p \omega_{k}(t)$.

It can be seen that value range of $f_{i}(t)$ is $[0,1]$. The value of $f_{i}(t)$ is more closer to 1 , the greater fairness of satisfaction user $i$ will get.

A novel cross-layer scheduling algorithm is proposed based on system performance joint optimization. The different system performance needs will be taken into consideration, the algorithm proposed in paper can endeavor to meet the QoS requirements of different types of cognitive users, and achieve good fair perception, make a good use of system bandwidth. At the same time, the algorithm can reduce power interference to authorized users caused by cognitive users obviously.

The cross-layer scheduling algorithm can be describe as follows:

$i^{*}=\underset{i}{\arg \max } \frac{\beta_{i} \times\left[R_{i}(t)\right]^{a_{1}} \times[A T I(t+1)]^{a_{2}} \times\left[f_{i}(t+1)\right]^{a_{3}}}{\left(I_{i} / I_{\max }\right)^{a_{4}}}$,

Where,

$$
I_{\max }=\max _{i}\left\{I_{i}\right\}, i=1,2, \ldots, K
$$

Where, $I_{i}$ is power interference to authorized users caused by cognitive users

$$
I_{i}=P_{i} \tilde{d}_{i}^{-\alpha}
$$

$P_{i}$ is cognitive users transmit power, $\tilde{d}_{i}$ is the distance between cognitive users $i$ and authorized users, $\alpha$ is power dissipation index.

$$
A T I(t)=\frac{1}{N} \sum_{i=1}^{N} T_{i} I_{i}(t)
$$

$\beta_{i}$ is the weight of different types of traffic, its value is as follows:

$$
\beta_{i}=\left\{\begin{array}{l}
\beta_{r t}, i \in R T \\
\beta_{n r t}, i \in N R T
\end{array}\right.
$$

Parameters $\beta_{i}$ is used to provide different types of traffic priorities. The user which has the higher priority will be scheduled earlier than the user which has lower priority.

$a_{1}, a_{2}, a_{3}, a_{4}$ are adjustable parameters, which are represent for index weighting of different factors in scheduling algorithm. As the use of index weighting, the system can quickly reach compromising between the different performances by adjusting the values of them.

\section{Simulation Analyses}

In the simulation, Six CRN users are taken into consideration, which including three real-time users and three non-real-time users. The six cognitive users use the same spectrum with an authorized user. The simulation parameters are shown in Table 1. Assumption that power interference range of the cognitive users caused to authorized user is $0-10 \mathrm{~dB}$, and they obey the same distribution. So the cognitive user who caused power interference is $0 \mathrm{~dB}$ to authorized user is the farthest one from authorized user, and the cognitive user who caused power interference is $10 \mathrm{~dB}$ to authorized user is the nearest one from authorized user.

Table 1 Simulation parameters.

\begin{tabular}{|l|c|c|c|c|c|c|c|}
\hline CID & $m$ & $\gamma$ & $\varepsilon_{i}$ & $D_{i}$ & $T_{i}$ & $C_{i}$ & $I_{i}$ \\
\hline 1 & 1.2 & 15 & $10^{-2}$ & 20 & 2.0 & 0.6 & 9.14 \\
2 & 1.0 & 20 & $10^{-2}$ & 40 & 1.0 & 0.5 & 8.44 \\
3 & 1.0 & 16 & $10^{-2}$ & 30 & 1.5 & 0.5 & 4.79 \\
4 & 1.0 & 15 & $10^{-2}$ & - & 5.0 & - & 10.0 \\
5 & 1.0 & 20 & $10^{-2}$ & - & 3.0 & - & 7.02 \\
6 & 1.0 & 18 & $10^{-2}$ & - & 4.0 & - & 0 \\
\hline
\end{tabular}

The algorithm mainly pays attention to power interference to authorized users caused by cognitive users, so in the simulation adjustable parameter $a_{1}, a_{2}, a_{3}$ are set as $0.01,1,1$. It analyzes the changes in system performance when $a_{4}$ are $0,0.5,1$. In the simulation, it will compare algorithm proposed with classical PFS algorithm. The classical PFS algorithm can be described as follows[14].

$$
i^{*}=\underset{i}{\arg \max } \frac{\beta_{i} \times R_{i}(t)}{f_{i}(t)},
$$

Latency default probability is used to describe latency performance of the proposed algorithm, and expression is as follows:

$$
E_{i}(t+1)=\left\{\begin{array}{l}
E_{i}(t)\left(1-1 / t_{c}\right), W_{i}(t)<D_{i} \\
E_{i}(t)\left(1-1 / t_{c}\right)+1 / t_{c}, W_{i}(t) \geqslant D_{i}, i \neq i^{*}
\end{array},\right.
$$

It only makes an example of user 1. Fig. 2 describes the latency default probability of real-time user 1. It can be seen from Fig. 2 that latency default probability of user 1 is less than $5 \%$ in most of the simulation time, which shows the algorithm provides a good delay guarantee for real-time business. The performance of real-time business has declined as $a_{4}$ gradually increasing, but real-time business also gets a good performance when $a_{4}=1$. At the same time, classical PFS algorithm also gets a good performance. 


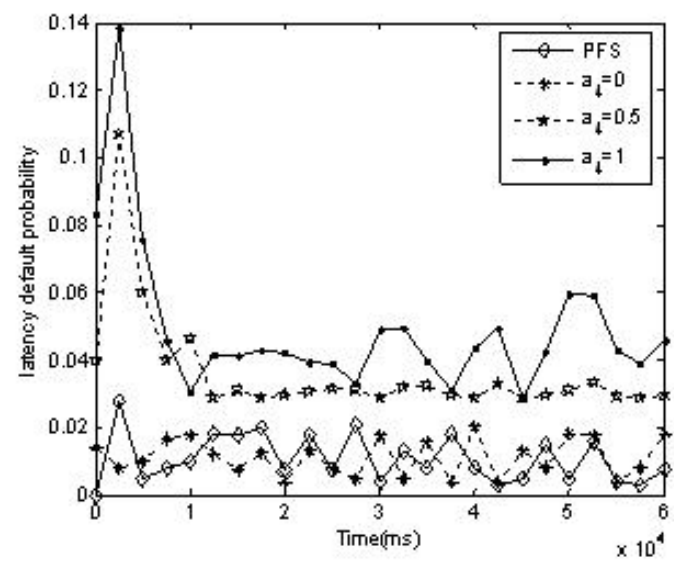

Fig. 2 Latency default probability of real-time user 1.

Fig.3 shows the throughput performance of non-real-time user 6 . The average transfer rate $T_{6}(t)$ of non-real time user 6 is greater than respective minimum guaranteed rate $T_{6}(t)=4$ by using the algorithm proposed in this paper. At the same time It can be seen that fluctuation of performance is very small. And it illustrates that the algorithm can easily meet requirements of minimum guarantee rate for non-real-time traffic. The power interference caused by cognitive users to authorize users plays a certain role in scheduling decision, but the QoS state, the channel conditions and other fairness limit factors also have a great impact on scheduling decision. So algorithm achieves a good performance when it makes good use of the multi-user diversity. The performance when $a_{4}=1$ is similar to $a_{4}=0.5$. It can be seen from Fig. 3 that throughput performance of user 6 has been significantly improved. It is because that power interference caused by user 6 is the smallest one. Performance of classical PFS algorithm is worst in Fig.3.

Fig.4 shows system fair performance curve, PFS algorithm obtains a good fairness performance because that its main objective is to ensure fairness between different users. The performance curve of proposed algorithm is similar to PFS algorithm when taking interference factors into consideration. Performance curve appear significant decline when $a_{4}=0$. It is because that $A T I(t)$ make real-time users obtain more chance for scheduling after minimum QoS requirements are need.

Fig. 5 describes the average power interference curve which the cognitive users to authorized users. Average power interference can be defined as follows[9].

$$
A I(t)=\frac{\sum_{j=1}^{t} I_{i}(t)}{t}
$$

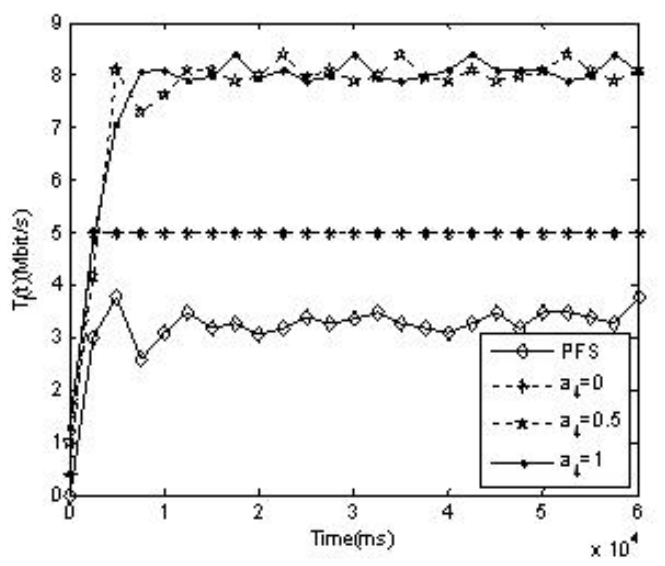

Fig. 3 Throughput performance of non-real-time user 6 .

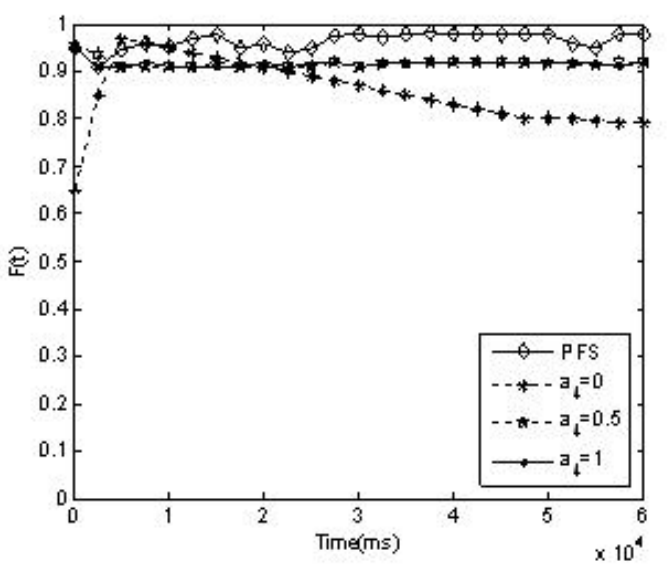

Fig. 4 Fair performance measures for system

In Fig.5, the algorithm proposed in this paper is significantly better than PFS algorithm. The algorithm proposed has more obvious advantages especially when the power interference becomes an important factor in scheduling guidelines. The performance when $a_{4}=0.5$ and $a_{4}=1$ is basally same.

It can be seen from the simulation results that the algorithm can efficiently schedule cognitive users. The power interference to authorized users caused by cognitive users also gets a better suppression. And the minimum requirements can easily be guaranteed. The algorithm achieves a good fairness performance between different types of users, at the same time it makes a full use of system bandwidth. And power interference to authorized users caused by cognitive users is reduced obviously. 


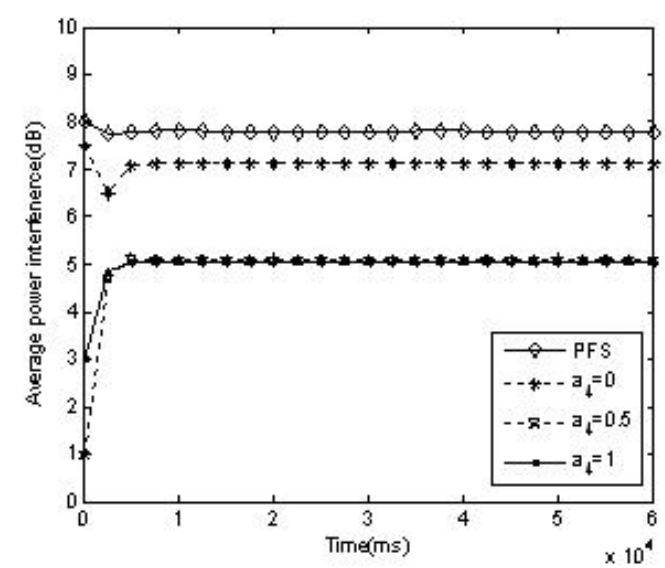

Fig. 5 Average power interference caused by cognitive network to authorized users under different scheduling algorithms

\section{Conclusions}

Development trend of wireless technology is proceeding along broadband, seamless and intelligent. A challenge that we must face is the lacking of spectrum resources. CRN can improve spectrum resource utilization rate, and it has a very broad application prospect[15]. At first, it makes a simple analysis of the definition and structure of $\mathrm{CRN}$, including the physical layer transmission mode, adaptive modulation and coding techniques, the power interference to authorized users caused by cognitive users and QoS requirements, etc. At the same time, a cross-layer algorithm is proposed. Simulation results show the algorithm can guarantee minimum QoS requirements of real-time traffic and non-real-time traffic by setting a different index weights. The algorithm provides a good fair performance, and can significantly reduce the average power interference to authorized users caused by cognitive users[16].

For next generation of high-speed broadband wireless communication systems, various kinds of interference errors and uncertainties factors are always present and will have an impact on system performance[17]. However, the cross-layer scheduling algorithm proposed in this paper is based on an ideal assumption. Such as the channel state is quasi-static, channel estimation is error-free, feedback channel is ideal and so on. Therefore, the study for cross-layer scheduling algorithm of non-ideal environment actual system will have more practical significance. And it will be one of the future research directions.

\section{References}

[1] Daeseob Lee, Dongho Won, A Study on Security Management Service System for Wireless Network Environment, Applied Mathematics \& Information Sciences, 6, 209-220 (2012)

[2] Mitola J., Maguire G. Q. JR., Cognitive radio: making software radios more personal, IEEE Personal Communications, 6, 13-18 (1999).

[3] M. V. Schaar, N. S. Shankar, Cross-layer wireless multimedia transmission: challenges, principles, and new paradigms, IEEE Wireless Communications, 12, 50-58 (2005).

[4] Le H.-S.T., Ly H.D. Opportunistic spectrum access using fuzzy logic for cognitive radio network, Communications and Electronics, 2008. Second International Conference on, Hoi an Vietnam, June, 240-245 (2008).

[5] Allen B. Mackenzie and Charles W. Bostion etc, Cognitive radio and networking research at Virginia Tech, Proceedings of the IEEE, 97, 660-688 (2009).

[6] Ahyoung Lee, Ilkyeun Ra. A Queuing Network Model Based on Ad Hoc Routing Networks for Multimedia Communications, Applied Mathematics \& Information Sciences, 6, 271-283 (2012)

[7] L. Hongyan, The research of key technologies for cognitive radio networks, Doctoral Dissertation, University of Posts and Telecommunication, Beijing, China, (2009).

[8] IEEE 802.16 Working Group. FEC Performance with ARQ and Adaptive Burst Profile Selection, (2001).

[9] Fortuna C, Mohorcic M, Trends in the Development of Communication Networks: Cognitive Networks, Computer Networks, 53, 1354-1376 (2009).

[10] O.S. Shin and K.B. Lee, Packet scheduling over a shared wireless link for heterogeneous classes of traffic, Proc. of IEEE International Conf. on Communication, Seoul Nat. Univ., South Korea, 58C62, (2004).

[11] Qingwen Liu, Shengli Zhou and Giannakis G.B., Crosslayer modeling of adaptive wireless links for QoS support in multimedia networks, Quality of Service in Hetergeneous Wired/wireless Networks, 2004, MN, USA Octber, 68-75 (2004).

[12] Haixia Zhang, Dongfeng Yuan and Yanbo Ma, Crosslayer Design for Wireless Communications-From Principle to Application, Posts and Telecom Press, Beijing, China, (2010).

[13] Yang Liu, Xinsheng Ji, Cross-layer packet scheduling scheme for supporting QoS of real-time services, Application Research of Computers, 28, 1810-1812 (2011).

[14] Tian Chong, Cross-layer scheduling algorithm based on wireless networks, doctoral diss., Shandong university, Jinan, China, (2009).

[15] Z. Ding, K. K. Leung, Cross-layer routing optimization for wireless networks with cooperative diversity, Personal, Indoor and Mobile Radio Communications, 2008, Cannes, French, September, 1-5 (2008).

[16] Qian Zhang, Juncheng Jia and Jin Zhang; Cooperative relay to improve diversity in cognitive radio networks, Communications Magazine, IEEE, 47, 111-117 (2009).

[17] Ning Zhang, Research on Control Routing Technology in Communication Network, Applied Mathematics \& Information Sciences, 6, 129-133 (2012). 


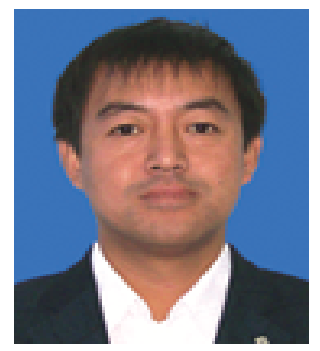

Changpeng Ji received the B.E. and M.S. degrees, in computer engineering from Liaoning Technical University of China, Fuxin, China, in 1993 and 2002. Professor of Liaoning Technical University, master instructor. The main research direction: computer communication \& networks, signal detection and estimation, wireless communication.

\section{Xiaoqiu}

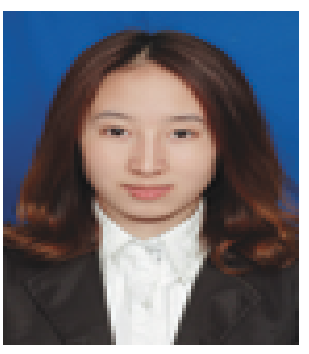

Nie received the bachelor degree in Communication Engineering from Liaoning Technical University, Liaoning, China, in 2010, and is currently studying towards the master degree at Liaoning Technical University. Her main research direction: wireless communication and

Cross-layer scheduling algorithm.

Yujie Yu received the bachelor degree in Communication Engineering from Liaoning Technical University, Liaoning, China, in 2010, and is currently studying towards the master degree at Liaoning Technical University. Her main research direction: signal processing. 